\title{
LACK OF INTEGRABILITY VIA VISCOSITY SOLUTION METHODS
}

\author{
DIOGO A. GOMES, ENRICO VALDINOCI
}

\begin{abstract}
In this paper we use viscosity solution methods to show that almost integrable Hamiltonian systems are not smoothly integrable at rotation numbers which are exponentially close to resonances.
\end{abstract}

\section{Contents}

1. Introduction 1

2. Viscosity Solutions 4

3. Estimates for the action $\quad 7$

4. Construction of Periodic Orbits 11

5. An explicit example $\quad 15$

References $\quad 16$

\section{INTRODUCTION}

A main problem in classical mechanics is to study the existence invariant tori for Hamiltonian systems of the form:

$$
H(p, x)=\frac{1}{2}|p|^{2}+\epsilon V(x),
$$

in which $V$ is a smooth $\mathbb{Z}^{N}$-periodic potential. The KAM theorem asserts that tori with frequencies $\Omega$ that satisfy a Diophantine condition:

$$
|\Omega \cdot k| \geq C|k|^{-\nu}, \quad \forall k \in \mathbb{Z}^{N}
$$

persist provided that $\epsilon$ is small enough. More precisely, KAM theory asserts that for all such $\Omega$ and sufficiently small $\epsilon$ there are smooth functions $f(X, \epsilon)=f(X, \epsilon ; V, \Omega)$ and $g(X, \epsilon)=g(X, \epsilon ; V, \Omega)$, periodic in $X$, such that, if we set

$$
x(t)=X(t)+f(X(t), \epsilon) \quad p(t)=g(X(t), \epsilon)
$$

We would like to thank Ugo Bessi for many enlightening discussions. 
with

$$
\dot{X}=\Omega,
$$

then $(x(t), p(t))$ is a solution to the Hamiltonian dynamics

$$
\dot{x}=-D_{p} H(p, x) \quad \dot{p}=D_{x} H(p, x) .
$$

Furthermore, $g$ is unique, and $f$ is determined up to a translation. Additionally, there is a vector $P$ and a number $\bar{H}(P)$ for which the Hamilton-Jacobi equation

$$
H\left(P+D_{x} u, x\right)=\bar{H}(P)
$$

has a unique (up to constants) smooth periodic solution and

$$
g(X, \epsilon)=P+D_{x} u(X+f(X, \epsilon)) .
$$

Since the curves $x(t)$ are characteristics to the Hamilton-Jacobi equation and smooth solutions are viscosity solutions, these curves are minimizers of the action

$$
\int_{0}^{T} L(x, \dot{x})+P \cdot \dot{x}+\bar{H}(P) .
$$

for all times $T$ among all curves with the same endpoints.

However, it is known that tori with frequencies that are sufficiently close to resonances can be destroyed by arbitrarily small perturbations (see, for instance, [Mat88], [Mat86], [For94], and [Bes00]), even if $V$ is analytic. The objective of this paper is to give an elementary proof that does not exist a change of coordinates as in (2) which satisfies very weak bounds.

We say that $H$ is $\alpha$-smoothly integrable at the rotation number $\Omega$ if:

(A) There is a smooth function:

$$
f(X, \epsilon)=f(X, \epsilon ; V, \Omega)
$$

periodic in $X$, and a differentiable function $u(x, \epsilon)$ such that for

$$
g(X, \epsilon)=P+D_{x} u(X+f(X, \epsilon), \epsilon)
$$

and any $x_{0} \in \mathbb{T}^{N}$,

$$
(x(t), p(t))=\left(x_{0}+\Omega t+f\left(x_{0}+\Omega t, \epsilon\right), g\left(x_{0}+\Omega t, \epsilon\right)\right)
$$

is a solution to the Hamiltonian dynamics. Also,

$$
\left\{x+f(x, \epsilon), x \in \mathbb{T}^{N}\right\}=\mathbb{T}^{N} \text {. }
$$


(B) $f$ satisfies the bound:

$$
\left|D_{X} f(X, \epsilon)\right|,\left|D_{X^{2}}^{2} f(X, \epsilon)\right| \leq C \epsilon^{\alpha},
$$

for a suitable $\alpha \in \mathbb{R}$ and a positive constant $C$, which may depend on $\Omega$ and $V$.

Hypothesis (A) means that we are looking for a Lagrangian torus that is a graph of a closed one-form $\left(x, P+D_{x} u\right)$, as the ones guaranteed by the KAM theory. The function $u$ is a $C^{1}$ solution to the HamiltonJacobi equation

$$
H\left(P+D_{x} u, x\right)=\bar{H}(P),
$$

and the orbit $x(t)$ in $(\mathbf{A})$, which is a characteristic for $u$, is is minimal, that is, it minimizes

$$
S_{x}(t)+u(x(t))
$$

among all Lipschitz curves with the same initial condition, in which $u$ is the viscosity solution of the Hamilton-Jacobi equation and

$$
S_{x}(T)=\int_{0}^{T} \frac{1}{2}|\dot{x}|^{2}-\epsilon V(x(t))+P \dot{x}+\bar{H}(P) d t .
$$

We say that the rotation number $\Omega$ is exponentially close to a resonance if there exists a sequence $T_{n} \rightarrow \infty$ and $\omega_{n} \in \frac{1}{T_{n}} \mathbb{Z}^{N}$ such that

$$
\left|\Omega-\omega_{n}\right| \leq e^{-b T_{n}} .
$$

In this paper we give an elementary proof that if $\Omega$ is exponentially close to a resonance, then it is possible to construct an analytic potential $V$ such that the Hamiltonian $H$ is not $\alpha$-smoothly integrable at $\Omega$, even for negative $\alpha$. In particular, Theorem 6 below will provide a necessary condition for smooth integrability (namely, estimate (8) below), which can be easily checked in explicit examples. For instance, in Theorem 8 an explicit analytic example violating such condition is dealt with.

Analogously, considering $C^{k}$ (instead of analytic potentials) one can also prove that $\alpha$-smooth integrability also fails if the rotation number $\Omega$ is polynomially close to resonances, that is:

$$
\left|\Omega-\omega_{n}\right| \leq \frac{C}{T_{n}^{\beta}},
$$

for large enough $\beta$.

We remark that, unlike the standard converse KAM methods, we do not rely on any computation of the splitting of separatrices and, in fact, 
our techniques are only based on a-priori estimates for viscosity solutions and minimizing orbits to the Lagrangian dynamics. Our proof, as the one in [Bes00], for instance, relies in showing that if such a torus exist then its orbits do not minimize the action. In [Bes00], this is done by computing the action along homoclinic orbits. In this paper, we use periodic orbits, constructed using elementary bifurcation theory, which are not minimizers of the action. By taking larger and larger periods we show that such a torus does not exit for arbitrarily small $\epsilon$. An important feature is that $\alpha$ in (3) is allowed to take negative values. Moreover, we can treat potentials with infinitely many harmonics (see Section 5).

The paper is organized as follows: in section 2 we recall the main facts and estimates on viscosity solutions for Hamilton-Jacobi equations; the main technical points, estimates for the action of minimizing orbits, and the construction of periodic orbits, using Liapunov-Schmidt reduction, are discussed, respectively, in sections 3 and 4; finally, an analytic potential $V$ for which $H$ is not $\alpha$-smoothly integrable is constructed in section 5 .

\section{Viscosity Solutions}

In this section we recall some facts about viscosity solutions of HamiltonJacobi equations. As stated in the introduction, such results will be the key to study the non-existence of smooth integrability. As most of these results are not new, they will be stated for the convenience of the reader, and the proofs can be found in the references mentioned.

The first result we quote is the classical Lions, Papanicolao and Varadhan theorem on the existence of periodic viscosity solutions to Hamilton-Jacobi equations [LPV88].

Theorem 1 (Lions, Papanicolao, Varadhan). Suppose

$$
H(p, x)=\frac{1}{2}|p|^{2}+\epsilon V(x)
$$

with $V(x)$ smooth and $\mathbb{Z}^{N}$ periodic in $x$. Then, for any value $P \in$ $\mathbb{R}^{N}$ there exists a unique number $\bar{H}(P)$, the effective Hamiltonian, for which the equation

$$
H\left(P+D_{x} u(x, P), x\right)=\bar{H}(P)
$$

has a $\mathbb{Z}^{N}$-periodic viscosity solution $u$. Furthermore $\bar{H}(P)$ is convex in $P$. The viscosity solution is semiconcave and Lipschitz. 
For the readers which are not familiar with viscosity solutions, we recall that a viscosity solution $u$ to (4) is a continuous function with the following property: for any smooth function $\phi$ and at any point $x_{0}$ at which $u-\phi$ has a local maximum (resp. local minimum) we have $H\left(P+D_{x} \phi\left(x_{0}\right), x_{0}\right) \leq \bar{H}(P)($ resp. $\geq \bar{H}(P))$.

Viscosity solutions of (4) have a variational characterization as fixed points of a control theory problem (see, for instance [FS93]). The action $S_{x}$ of a Lipschitz orbit $x(t)$ is given by

$$
S_{x}(T)=\int_{0}^{T} \frac{1}{2}|\dot{x}|^{2}-\epsilon V(x(t))+P \dot{x}+\bar{H}(P) d t .
$$

Then, we have:

Theorem 2. Let $u$ be a viscosity solution to (4) and $S_{x}$ as in (5). Then,

$$
u(x)=\inf _{x(\cdot), x(0)=x} S_{x}(T)+u(x(T)),
$$

in which the infimum is taken over all Lipschitz trajectories $x(\cdot)$ with initial condition $x(0)=x$. Furthermore, there is a minimizing trajectory which has $\|\dot{x}\|_{L^{\infty}} \leq C$, independent of $\epsilon$, for small $\epsilon$.

In general, the solutions to (4) are not smooth, however viscosity solutions enjoy some regularity, as shown in the next theorem.

Lemma 1 (A priori Lipschitz estimates). Suppose $u$ is a viscosity solution to (4). Then, for any $h$

$$
u(x+h)+u(x-h)-2 u(x) \leq C\|D V\|_{L^{\infty}} \epsilon^{1 / 2}\|h\|^{2},
$$

in which $\|\cdot\|$ is the periodic distance in the torus:

$$
\|z\|=\inf _{k \in \mathbb{Z}^{N}}|z+k|
$$

In particular, this implies

$$
\|D u\|_{L^{\infty}} \leq C\|D V\|_{L^{\infty}} \epsilon^{1 / 2}
$$

Proof. Since $u$ is periodic, we may assume that $\|h\|=|h|$. Let $x(t)$, $0 \leq t \leq \frac{1}{\sqrt{\epsilon}}$, be an optimal trajectory with $x(0)=x$. Define

$$
y^{ \pm}(t)=x(t) \pm h(1-\sqrt{\epsilon} t) .
$$


Then

$$
\begin{aligned}
& u(x+h)+u(x-h)-u(x) \leq \\
& \leq \int_{0}^{1 / \sqrt{\epsilon}} \frac{1}{2}|\dot{x}(t)-\sqrt{\epsilon} h|^{2}+\frac{1}{2}|\dot{x}(t)+\sqrt{\epsilon} h|^{2}-|\dot{x}(t)|^{2}+ \\
& \quad+O\left(\epsilon\|D V\|_{L^{\infty}}|h|\right) d t \leq \\
& \leq C\|D V\|_{L^{\infty} \sqrt{\epsilon}|h| .}
\end{aligned}
$$

It is a well know result that a semiconcave periodic function is Lipschitz.

Since the works of Fathi [Fat97a, Fat97b, Fat98a, Fat98b], and E [E99], among others, the connection between viscosity solutions and the weak KAM theory has become a well established fact. We summarize some of these results in the next theorem (see also [EG01]).

Theorem 3. Let $u(x, P)$ be a viscosity solution to (4). Then, for any $P$ there exists a set, the Mather set, contained in the graph

$$
\left(x, P+D_{x} u(x, P)\right),
$$

which is invariant under the Hamiltonian dynamics

$$
\dot{x}=-D_{p} H(p, x) \quad \dot{p}=D_{x} H(p, x) .
$$

Furthermore, if $u$ is smooth, this set is an invariant torus and any trajectory $(x(\cdot), p(\cdot))$ to the Hamiltonian dynamics is a minimizer, that is

$$
u(x(0))=S_{x}(T)+u(x(T)) .
$$

The rotation vector $\omega$ of a curve $x(\cdot)$ is the limit

$$
\lim _{T \rightarrow \infty} \frac{x(T)}{T}=\omega
$$

provided it exists. It turns out that one can relate the differentiability properties of the effective Hamiltonian $\bar{H}$ with asymptotic properties, such as the rotation number, of the trajectories in the Mather set. In the next theorem we state one possible result, see also [Gom02] for sharper asymptotic estimates.

Theorem 4. Suppose that $x(\cdot)$ is a minimizing orbit corresponding to some $P$. Then, the rotation number $\omega$ of $x(\cdot)$ is

$$
\omega=-D_{P} \bar{H}(P)
$$

provided $\bar{H}(P)$ is differentiable at $P$. 
Let $L(x, v)$ be the Legendre transform of $H(p, x)$, that is,

$$
L(x, v)=\sup _{p}-p \cdot v-H(p, x) .
$$

In our case

$$
L(x, v)=\frac{1}{2}|v|^{2}-\epsilon V(x) .
$$

The Legendre transform of $\bar{H}(P)$ is defined by

$$
\bar{L}(\omega)=\sup _{P}-P \cdot \omega-\bar{H}(P) .
$$

The next theorem shows that $\bar{L}$ is an average of $L$ along a minimizing trajectory [EG01].

Theorem 5. Let $x(\cdot)$ be a minimizing trajectory with rotation vector $\omega$, then,

$$
\bar{L}(\omega)=\lim _{T \rightarrow \infty} \frac{1}{T} \int_{0}^{T} L(x, \dot{x}) d t .
$$

In particular, if the supremum on (6) is achieved at $P^{\omega}$, then

$$
\bar{L}(\omega)+\bar{H}\left(P^{\omega}\right)+P^{\omega} \cdot \omega=0 .
$$

\section{Estimates FOR THE ACTION}

In this section we prove the main technical estimates concerning the difference of action between orbits of different rotation numbers. All these estimates are elementary, but they are also the key to our main result.

In what follows, we assume the frequency $\Omega$ to be fixed.

In the first lemma, we consider two different periodic orbits, not necessarily minimizing nor satisfying the equations of motion, with the same period $T$ and rotation vector $\omega$. In general, they will have different action, which can be estimated, for small $\epsilon$, by the Melnikov gap:

$$
M=\int_{0}^{T} V\left(\bar{x}_{0}+\omega t\right)-V\left(\bar{x}_{1}+\omega t\right) d t .
$$

Lemma 2. Let $x_{0}$ and $x_{1}$ be two periodic orbits in $\mathbb{T}^{N}$ of frequency $\omega \in \frac{1}{T} \mathbb{Z}^{N}$. Assume that

$$
x_{i}(t)=\bar{x}_{i}+\omega t+\epsilon y_{i}(t)
$$


with $y_{i}(0)=y_{i}(T)$. Assume further that $y_{i}$ together with its derivative are bounded. Then,

$$
S_{x_{0}}(T)-S_{x_{1}}(T) \geq \epsilon \int_{0}^{T} V\left(\bar{x}_{1}+\omega t\right)-V\left(\bar{x}_{0}+\omega t\right) d t-O\left(\epsilon^{2} T\right) .
$$

Furthermore, the term $O\left(\epsilon^{2} T\right)$ can be estimated by

$$
C \epsilon^{2} T\left[\|\dot{y}\|_{L^{\infty}}^{2}+\|D V\|_{L^{\infty}}\|y\|_{L^{\infty}}\right] .
$$

Proof. Observe that

$$
\begin{aligned}
S_{x_{0}}(T)-S_{x_{1}}(T)= & \int_{0}^{T} \frac{1}{2}\left|\omega+\epsilon \dot{y}_{0}\right|^{2}-\frac{1}{2}\left|\omega+\epsilon \dot{y}_{1}\right|^{2}+ \\
& +\epsilon V\left(\bar{x}_{1}+\omega t+\epsilon y_{1}\right)-\epsilon V\left(\bar{x}_{0}+\omega t+\epsilon y_{0}\right)+ \\
& +P^{\omega} \cdot\left(\omega+\epsilon \dot{y}_{0}\right)-P^{\omega} \cdot\left(\omega+\epsilon \dot{y}_{1}\right) d t \geq \\
\geq & \epsilon \int_{0}^{T} V\left(\bar{x}_{1}+\omega t\right)-V\left(\bar{x}_{0}+\omega t\right) d t-O\left(\epsilon^{2} T\right)
\end{aligned}
$$

In the next lemma, we consider orbits whose rotation numbers are close and have the same initial point, and estimate the difference between their actions. For simplicity, in what follows we will assume $T \geq 1$.

Lemma 3. Let $\omega$ and $\Omega$ be given rotation numbers. Assume that $x(t)$ and $z(t)$ are, respectively, orbits of rotation numbers $\omega$ and $\Omega$. Suppose that $x(t)$ has period $T$ and minimizes the action among all $T$-periodic orbits with initial condition $x(0)$. Furthermore assume that

$$
z(t)=\Omega t+f(\Omega t, \epsilon)
$$

satisfies $x(0)=z(0)$. Then

$$
S_{x}-S_{z} \leq C_{a} T^{2}|\omega-\Omega|,
$$

the constant $C_{a}$ satisfies:

$$
C_{a} \leq C\left(1+\|D f\|_{L^{\infty}}+\left\|D^{2} f\right\|_{L^{\infty}}+\|D V\|_{L^{\infty}}\right)^{2}
$$

Proof. Since the orbit $x(t)$ is minimal, the orbit $\tilde{x}=\omega t+f(\omega t, \epsilon)$ has a larger action, that is:

$$
\begin{aligned}
S_{x}(T) \leq & \int_{0}^{T} \frac{1}{2}|\omega+D f(\omega t, \epsilon) \omega|^{2}-\epsilon V(\omega t+f(\omega t, \epsilon))+ \\
& +P^{\omega} \cdot D f(\omega t, \epsilon) \omega-\bar{L}(\omega) d t
\end{aligned}
$$


using the fact that, for any $\omega$,

$$
\bar{H}\left(P^{\omega}\right)+\bar{L}(\omega)+P^{\omega} \cdot \omega=0
$$

Furthermore

$$
\begin{aligned}
S_{z}(T)= & \int_{0}^{T} \frac{1}{2}|\Omega+D f(\Omega t, \epsilon) \Omega|^{2}-\epsilon V(\Omega t+f(\Omega t, \epsilon))+ \\
& +P^{\Omega} \cdot D f(\Omega t, \epsilon) \Omega-\bar{L}(\Omega) d t
\end{aligned}
$$

Then, since $\bar{L}$ is convex it is locally Lipschitz, and so

$$
\int_{0}^{T} \bar{L}(\omega)-\bar{L}(\Omega) \leq C|\omega-\Omega| T .
$$

Since $f(\omega, T, \epsilon)=f(0, \epsilon)$, we have

$$
\begin{aligned}
& \int_{0}^{T}\left[P^{\omega} \cdot D f(\omega t, \epsilon) \omega-P^{\Omega} \cdot D f(\Omega t, \epsilon) \Omega\right]= \\
& =-P^{\Omega}[f(\Omega T, \epsilon)-f(0, \epsilon)] \\
& \quad \leq C_{0}|\Omega-\omega| T
\end{aligned}
$$

and the constant $C_{0}$ can be estimated by $C_{0} \leq C\left(1+\|D f\|_{L^{\infty}}\right)$.

The remaining terms can be estimated by:

$$
\begin{aligned}
& \int_{0}^{T} \frac{1}{2}|\omega+D f(\omega t, \epsilon) \omega|^{2}-\frac{1}{2}|\Omega+D f(\Omega t, \epsilon) \Omega|^{2}- \\
& \quad-\epsilon V(\omega t+f(\omega t, \epsilon))+\epsilon V(\Omega t+f(\Omega t, \epsilon)) d t \leq \\
& \quad \leq C_{1} T^{2}|\omega-\Omega|,
\end{aligned}
$$

the constant $C_{1}$ can be bounded by

$$
C_{1} \leq C\left(1+\|D f\|_{L^{\infty}}+\left\|D^{2} f\right\|_{L^{\infty}}+\|D V\|_{L^{\infty}}\right)^{2}
$$

Lemma 4. For any $T$-periodic curve $x(t)$ with rotation number $\omega$ we have

$$
S_{x}(T) \geq 0
$$

Proof. Recall that

$$
S_{x}(T)=\int_{0}^{T} L(x, \dot{x})+P^{\omega} \cdot(\dot{x}-\omega)-\bar{L}(\omega) d t
$$


and

$$
\begin{aligned}
\bar{L}(\omega) & \leq \lim _{n \rightarrow \infty} \frac{1}{n T} \int_{0}^{n T} L(x, \dot{x})+P^{\omega} \cdot(\dot{x}-\omega) d t= \\
& =\frac{1}{T} \int_{0}^{T} L(x, \dot{x})+P^{\omega} \cdot(\dot{x}-\omega) d t .
\end{aligned}
$$

We show in the next lemma that the action $S$ of a minimizing curve is almost independent on the initial point.

Lemma 5. Suppose that the system is smoothly integrable at $\Omega$ and let $z=\Omega t+f(\Omega t, \epsilon)$. Then,

$$
\left|S_{z}(T)\right| \leq C \epsilon^{1 / 2}\|z(T)-z(0)\|,
$$

in which $\|\cdot\|$ is the periodic distance in the torus. Furthermore, if $\omega \in \frac{1}{T} \mathbb{Z}^{N}$, then

$$
\left|S_{z}(T)\right| \leq C_{1} \epsilon^{1 / 2}|\omega-\Omega| T
$$

and the constant $C_{1} \leq C\left(1+\|D f\|_{L^{\infty}}\right)\left(1+\|D V\|_{L^{\infty}}\right)$.

ProOF. The first estimate follows from the existence of a $\left(C^{1}\right)$ viscosity solution $u$ corresponding to $\Omega$, from (A) at page 2 , the a-priori Lipschitz estimates of lemma 1 , and

$$
S_{z}(T)=u(z(0))-u(z(T)) .
$$

The second estimate follows from the first one and the hypothesis.

By combining all the previous lemmas, one can prove that if the Melnikov gap $M$, as defined in (7), is too large, then the system is not smoothly integrable at $\Omega$.

Theorem 6. Suppose the system is smoothly integrable at $\Omega$ and assume that there are two periodic orbits $x_{0}, x_{1}$, with period $T$, with frequency $\omega \in \frac{1}{T} \mathbb{Z}^{N}$, of the form

$$
x_{i}(t)=\bar{x}_{i}+\omega t+\epsilon y_{i}(t),
$$

as in lemma 2. Let us assume that $x_{i}$ are action minimizing among all $T$-periodic orbits with initial condition $x_{i}(0)$. Let

$$
M=\int_{0}^{T} V\left(\bar{x}_{1}+\omega t\right)-V\left(\bar{x}_{0}+\omega t\right) d t .
$$

Then,

$$
M \leq\left[C_{1} \frac{T^{2}}{\epsilon}|\omega-\Omega|+C_{2} \epsilon T\right] .
$$


The constant $C_{1}$ depends polynomially in $\|D f\|_{L^{\infty}},\left\|D^{2} f\right\|_{L^{\infty}},\|D V\|_{L^{\infty}}$ and $\|V\|_{L^{\infty}}$; the constant $C_{2}$ depends polynomially in $\left\|D^{2} V\right\|_{L^{\infty}},\|D V\|_{L^{\infty}}$, $\|V\|_{L^{\infty}}$, in $\left\|y_{i}\right\|_{L^{\infty}},\left\|\dot{y}_{i}\right\|_{L^{\infty}}$.

PROOF. Let $z_{i}$ be a minimal orbit with frequency $\Omega$ of the form (2), with initial condition $\bar{z}_{i}=\bar{x}_{i}$. This choice is possible by assumption (A). Assume $S_{x_{0}} \leq S_{x_{1}}$. Then (evaluating all actions at time $T$ )

$$
S_{x_{1}}-S_{x_{0}} \leq S_{x_{1}}-S_{z_{1}}+S_{z_{1}}-S_{x_{0}} .
$$

From lemma 2, we have

$$
\epsilon M-C \epsilon^{2} T \leq S_{x_{1}}-S_{x_{0}} .
$$

Lemma 3 yields

$$
S_{x_{1}}-S_{z_{1}} \leq C_{a} T^{2}|\omega-\Omega|
$$

Lemma 5 implies

$$
\left|S_{z_{1}}\right| \leq C_{1} \epsilon^{1 / 2}|\omega-\Omega| T
$$

Finally, from lemma 4

$$
S_{x_{0}} \geq 0
$$

yields the last inequality necessary to the estimate.

\section{Construction of Periodic Orbits}

The main goal of this section is to use a well known LiapunovSchmidt reduction to construct periodic orbits for which the estimates in the previous section can be applied.

Theorem 7. Let $\omega \in \frac{1}{T} \mathbb{Z}^{N}$. There exists $\Lambda>1$, polynomially depending on $T$ and on the $C^{3}$-norm of $V$ so that: if $\Lambda \epsilon<1$, the system has a periodic orbit $\left(x_{\omega}(t), p_{\omega}(t)\right)$ of rotation number $\omega$. Furthermore, such orbit has the form

$$
\begin{aligned}
& x_{\omega}(t)=x_{*}(0)+\omega t+\epsilon y_{\omega}(t), \\
& p_{\omega}(t)=\omega+\epsilon \dot{y}_{\omega}(t)
\end{aligned}
$$

with

$$
\begin{aligned}
& y_{\omega}(0)=y_{\omega}(T)=0, \\
& \sup _{t \in[0, T]}\left|y_{\omega}(t)\right|+T \sup _{t \in[0, T]}\left|\dot{y}_{\omega}(t)\right| \leq \Lambda .
\end{aligned}
$$


Proof. For the proof of this theorem we will follow the well known Liapunov-Schmidt reduction (see, for instance [ACZE87], [BBV03] and references therein). Remarkably, the desired periodic orbits will be obtained as critical points of a suitably restricted action functional. We first build a family of pseudo-orbits, that is, curves $(x(\cdot), p(\cdot))$ which satisfy the equations of motion and with $x(0)=x(T)$ and $p(0)=$ $p(T)+O(\epsilon)$.

Lemma 6. Let $\omega \in \frac{1}{T} \mathbb{Z}^{N}$. Fix $x_{*} \in \mathbb{T}^{N}$. Then, there exists $\Lambda>1$, polynomially depending on $T$ and on the $C^{3}$-norm of $V$ so that: if $\Lambda \epsilon<1$, there exists $\left(x_{x_{*}}(t), p_{x_{*}}(t)\right)$, whose dependence on $x^{*}$ is $C^{1}$, which satisfies the equation of motion for any time $t \in[0, T]$, and such that

$$
\begin{aligned}
& x_{x_{*}}(t)=x_{*}+\omega t+\epsilon y(t), \\
& p_{x_{*}}(t)=\omega+\epsilon \dot{y}(t), \\
& y(0)=y(T)=0, \\
& \sup _{t \in[0, T]}|y(t)|+T \sup _{t \in[0, T]}|\dot{y}(t)| \leq \Lambda .
\end{aligned}
$$

Proof. Let $X=C^{1}\left([0, T], \mathbb{R}^{N}\right), Y=\{f \in X: f(0)=f(T)=0\}$. Consider $X$ and $Y$ as Banach spaces endowed with the norm:

$$
\|f\|=\sup _{[0, T]}|f|+T \sup _{[0, T]}|\dot{f}| \text {. }
$$

Define the linear operator $\mathcal{L}$ on $Y$ as

$$
\mathcal{L} f(t)=\int_{0}^{t} \int_{0}^{\tau} f(s) d s d \tau-\frac{t}{T} \int_{0}^{T} \int_{0}^{\tau} f(s) d s d \tau, \quad \forall t \in[0, T] .
$$

Then, $\mathcal{L}(X) \subseteq Y$ and the operator norm of $\mathcal{L}$ is bounded by $4 T^{2}$. Furthermore, if $g=\mathcal{L} f$, then $g$ is $C^{2}$ and $\ddot{g}=f$. Additionally, let $\mathcal{P}$ be given by:

$$
\begin{gathered}
\mathcal{P}(f(t))=V^{\prime}\left(x_{*}+\omega t\right)-V^{\prime}\left(x_{*}+\omega t-\epsilon \mathcal{L}\left(V^{\prime}\left(x_{*}+\omega t\right)\right)+\epsilon f(t)\right)= \\
=V^{\prime}\left(x_{*}+\omega t\right)-V^{\prime}\left(x_{*}+\omega t+\epsilon \frac{t}{T} \int_{0}^{T} \int_{0}^{\tau} V^{\prime}\left(x_{*}+\omega s\right) d s d \tau-\right. \\
\left.\quad-\epsilon \int_{0}^{t} \int_{0}^{\tau} V^{\prime}\left(x_{*}+\omega s\right) d s d \tau+\epsilon f(t)\right) .
\end{gathered}
$$

Notice that $\|\mathcal{P}(0)\| \leq \Lambda \epsilon$, for a suitable constant $\Lambda$ which is polynomial in $T$ and in the $C^{3}$-norm of $V$. Moreover, the Lipschitz norm of $\mathcal{P}$ in the unit ball is bounded by $C \Lambda \epsilon$. Whence, $|\mathcal{L P}(0)| \leq C \Lambda \epsilon$, therefore 
(see [BBV03]), if (by changing $\Lambda \rightarrow C \Lambda$, if necessary) $\Lambda \epsilon<1$, there exists $g \in X$ so that $\|g\| \leq \Lambda$ and $g=\mathcal{L} \mathcal{P} g$. The desired pseudo-orbit is then

$$
\begin{aligned}
& x_{x_{*}}(t)=x_{*}+\omega t+\epsilon y(t), \\
& p_{x_{*}}(t)=\omega+\epsilon \dot{y}(t),
\end{aligned}
$$

where

$$
y(t)=\frac{t}{T} \int_{0}^{T} \int_{0}^{\tau} V^{\prime}\left(x_{*}+\omega s\right) d s d \tau-\int_{0}^{t} \int_{0}^{\tau} V^{\prime}\left(x_{*}+\omega s\right) d s d \tau+g(t) .
$$

Also, the map $x^{*} \mapsto\left(x_{x_{*}}, p_{x_{*}}\right)$ is $C^{1}$ by the standard Implicit Function Theorem.

The proof of theorem 7 ends as follows. Let $\left(x_{x_{*}}, p_{x_{*}}\right)$ be the pseudoorbit constructed above.

Define the functional $\mathcal{E}$ on $\mathbb{T}^{N}$ as

$$
\mathcal{E}\left(x_{*}\right)=\int_{0}^{T} L\left(x_{x_{*}}(t), \dot{x}_{x_{*}}(t)\right) d t .
$$

Then, critical points for $\mathcal{E}$ are periodic solutions of the equation of motion, as the reader may easily check by inspecting the Euler equation for $\mathcal{E}$. The existence of critical points for $\mathcal{E}$ on $\mathbb{T}^{N}$ is now a well known fact from finite-dimensional analysis.

Let us point out some easy relations between the functional $\mathcal{E}$ above and the action:

Lemma 7. If $\left(x_{x_{*}}(t), p_{x_{*}}(t)\right)$ is a pseudo-orbit constructed in lemma 6 and $\mathcal{E}$ is as in (9), then

$$
\left.\left|\mathcal{E}\left(x_{*}\right)+\epsilon \int_{0}^{T} V\left(x_{*}+\omega t\right) d t-\frac{T}{2}\right| \omega\right|^{2} \mid \leq \Lambda \epsilon^{2},
$$

with $\Lambda>1$ polynomially depending on $T$ and on the $C^{3}$-norm of $V$.

PROOF. It is a straightforward calculation, by using the estimates on the pseudo-orbit given in lemma 6 .

Corollary 1. Let the hypotheses of theorem 7 hold. Define, for any $x \in \mathbb{T}^{N}$,

$$
\mathcal{M}(x)=\int_{0}^{T} V(x+\omega t) d t .
$$

Let $x_{M}$ and $x_{m}$ be points of absolute maximum and minimum for $\mathcal{M}$, respectively. Let us assume that they are non-degenerate, i.e., that there 
exist positive quantities $\delta$ and $\mu$ so that

$$
\mathcal{M}\left(x_{M}\right) \geq \mathcal{M}(x)+\mu, \quad \forall x \in \partial B_{\delta}\left(x_{M}\right),
$$

and

$$
\mathcal{M}(x) \geq \mathcal{M}\left(x_{m}\right)+\mu, \quad \forall x \in \partial B_{\delta}\left(x_{m}\right) .
$$

Let also $m=\mathcal{M}\left(x_{M}\right)-\mathcal{M}\left(x_{m}\right)$. Then ${ }^{1}$, there exists $\Lambda>1$, polynomially depending on $T$ and on the $C^{3}$-norm of $V$ so that: if $\mu^{*}:=$ $\min \{\mu, 1\}$ and $\epsilon<\frac{\mu^{*}}{\Lambda}$, then $\mathcal{E}$ has at least one critical point $x_{M}^{*}$ in $B_{\delta}\left(x_{M}\right)$ and at least one critical point $x_{m}^{*}$ in $B_{\delta}\left(x_{m}\right)$. These critical points correspond to two different periodic orbits $x_{\omega, M}(t)$ and $x_{\omega, m}(t)$, of frequency $\omega$, whose action satisfies

$$
\left|S_{x_{\omega, M}}(T)-S_{x_{\omega, m}}(T)\right| \geq \epsilon \frac{m}{2} .
$$

ProOF. It follows directly from theorem 7, lemma 7 and the definition of the action.

We now point out that, even if one of the orbits constructed above maximizes the functional $\mathcal{E}$, they both minimize the action among all $T$-periodic orbits with the same initial point:

Lemma 8. For $\epsilon$ sufficiently small, the orbits $x(t)$ constructed above are minimizers of the action among all T-periodic orbits $\gamma(t)$ with $\gamma(0)=x(0)$.

Proof. We have

$$
\begin{aligned}
\int_{0}^{T} \frac{1}{2}|\dot{\gamma}|^{2}+\epsilon V(\gamma) d t \geq & \int_{0}^{T} \frac{1}{2}|\dot{x}|^{2}+\dot{x} \cdot(\dot{\gamma}-\dot{x})+\frac{1}{2}|\dot{x}-\dot{\gamma}|^{2}+ \\
& +\epsilon V(x)+\epsilon D_{x} V(x)(\gamma-x)-\epsilon C|\gamma-x|^{2} d t
\end{aligned}
$$

Since $x$ is a solution to the Euler-Lagrange equation we have

$$
\int_{0}^{T} \dot{x} \cdot(\dot{\gamma}-\dot{x})+\epsilon D_{x} V(x)(\gamma-x) d t=0 .
$$

Poincaré inequality applied to the interval $[0, T]$ yields

$$
\int_{0}^{T}|\gamma-x|^{2} \leq C \int_{0}^{T}|\dot{\gamma}-\dot{x}|^{2}
$$

Thus, for sufficiently small $\epsilon$ we have

$$
\int_{0}^{T} \frac{1}{2}|\dot{\gamma}|^{2}+\epsilon V(\gamma) d t \geq \int_{0}^{T} \frac{1}{2}|\dot{x}|^{2}+\epsilon V(x) d t .
$$

\footnotetext{
${ }^{1}$ Of course, $m \geq \mu>0$.
} 


\section{An explicit exAmple}

In this last section we construct an analytic potential for which $H$ is not smoothly integrable at rotation number $\Omega$, provided $\Omega$ is exponentially close to resonances. With minor modifications, it is possible to construct $C^{k}$ potentials for which the system is not smoothly integrable at a rotation number polynomially close to resonances.

Theorem 8. Let $N \geq 2$. Suppose the Hamiltonian $H$ is as in (1), and the rotation number $\Omega$ is irrational, that is, $\Omega \cdot k \neq 0$ for any $k \in \mathbb{Z}^{N}$, and exponentially close to a resonance, that is, there exists a sequence $T_{n} \rightarrow \infty$ and $\omega_{n} \in \frac{1}{T_{n}} \mathbb{Z}^{N}$ such that

$$
\left|\Omega-\omega_{n}\right| \leq e^{-b T_{n}} .
$$

Then, if $b$ is sufficiently large, there exists a periodic analytic potential $V$ for which $H$ is not $\alpha$-smoothly integrable at the rotation number $\Omega$.

PROOF. Let $\omega_{n}^{\prime}$ and $\Omega^{\prime}$ be, respectively, the projections of $\omega_{n}$ and $\Omega$ onto their first two components. Note that, taking a subsequence, if necessary, we may assume that none of the projections $\omega_{n}^{\prime}$ are parallel. Indeed, let us assume that, inductively, we constructed a set of rational frequencies

$$
\omega_{n_{1}}, \ldots, \omega_{n_{k}}
$$

so that $\omega_{n_{i}}^{\prime}$ and $\omega_{n_{j}}^{\prime}$ are non-parallel for each $i, j=1, \ldots, k$. Then, there must be an index $n^{*}>n_{k}$ so that $\omega_{n^{*}}^{\prime}$ is parallel to none of the $\omega_{n_{i}}^{\prime}$. Indeed, if not, there is a subsequence which is parallel to some $\omega_{n_{i_{0}}}^{\prime}$; since such subsequence converges to $\Omega^{\prime}$ and $\omega_{n_{i_{0}}}^{\prime}$ is rational, we would infer that $\Omega^{\prime}=\frac{1}{\tau}\left(\nu_{1}, \nu_{2}\right)$, for suitable $\nu_{1}, \nu_{2} \in \mathbb{Z}, \tau \in \mathbb{R} \backslash\{0\}$. Hence, $\Omega \cdot\left(-\nu_{2}, \nu_{1}, 0, \ldots, 0\right)=0$, in contradiction with the assumptions.

Thus, we now assume that none of the projections $\omega_{n}^{\prime}$ are parallel. Let us write $\omega_{n}^{\prime}=\frac{1}{T_{n}}\left(\nu_{n 1}, \nu_{n 2}\right)$, with $\nu_{n 1}, \nu_{n 2} \in \mathbb{Z}$. Consider a sequence $k_{n}=\left(-\nu_{n 2}, \nu_{n 1}, 0, \ldots, 0\right) \in \mathbb{Z}^{N}$. Then, $\left|k_{n}\right| \leq \operatorname{const} T_{n}, \omega_{n} \cdot k_{n}=0$, and $\omega_{i} \cdot k_{n} \neq 0$ if $n \neq i$. Therefore,

$$
\int_{0}^{T_{j}} \cos \left(2 \pi k_{n} \cdot\left(\bar{x}+\omega_{j} t\right)\right) d t=\delta_{n j} T_{n} \cos \left(2 \pi k_{n} \cdot \bar{x}\right) .
$$

Let

$$
V(x)=\sum_{n=1}^{\infty} e^{-c T_{n}} \cos \left(2 \pi k_{n} \cdot x\right)
$$


for some $c>0$ sufficiently large to make $V$ analytic.

We will show that for a sequence $\epsilon_{n} \rightarrow 0$ the inequality (8) in theorem 6 is not satisfied.

Fix $\omega_{n}$ and choose $\epsilon_{n}=e^{-d T_{n}}$, for some $d>0$ to be determined. By corollary 1 and lemma 8, we obtain two periodic orbits of the system, which are action minimizing among trajectories with their same initial point, and whose Melnikov gap is, at least, of the order of $T_{n} e^{-c T_{n}}$. Then, by means of (3), inequality (8) reads

$$
e^{-c T_{n}} \leq K_{1} T_{n}^{K_{2}}\left(\frac{\left|\Omega-\omega_{n}\right|}{\epsilon_{n}^{K_{3}}}+\epsilon_{n}\right) \leq K_{1} T_{n}^{K_{2}}\left(e^{\left(d K_{3}-b\right) T_{n}}+e^{-d T_{n}}\right),
$$

for suitable positive constants $K_{i}$. Therefore, if $d>c$ and $b>c+K_{3} d$, this inequality fails as $T_{n} \rightarrow \infty$.

\section{REFERENCES}

[ACZE87] A. Ambrosetti, V. Coti Zelati, and I. Ekeland. Symmetry breaking in Hamiltonian systems. J. Differential Equations, 67(2):165-184, 1987.

[BBV03] Massimiliano Berti, Luca Biasco, and Enrico Valdinoci. Abundance of periodic orbits in Hamiltonian systems with elliptic invariant tori and applications to the planetary three-body problem. preprint, 2003.

[Bes00] Ugo Bessi. An analytic counterexample to the KAM theorem. Ergodic Theory Dynam. Systems, 20(2):317-333, 2000.

[E99] Weinan E. Aubry-Mather theory and periodic solutions of the forced Burgers equation. Comm. Pure Appl. Math., 52(7):811-828, 1999.

[EG01] L. C. Evans and D. Gomes. Effective Hamiltonians and averaging for Hamiltonian dynamics. I. Arch. Ration. Mech. Anal., 157(1):1-33, 2001.

[Fat97a] Albert Fathi. Solutions KAM faibles conjuguées et barrières de Peierls. C. R. Acad. Sci. Paris Sér. I Math., 325(6):649-652, 1997.

[Fat97b] Albert Fathi. Théorème KAM faible et théorie de Mather sur les systèmes lagrangiens. C. R. Acad. Sci. Paris Sér. I Math., 324(9):10431046, 1997.

[Fat98a] Albert Fathi. Orbite hétéroclines et ensemble de Peierls. C. R. Acad. Sci. Paris Sér. I Math., 326:1213-1216, 1998.

[Fat98b] Albert Fathi. Sur la convergence du semi-groupe de Lax-Oleinik. C. $R$. Acad. Sci. Paris Sér. I Math., 327:267-270, 1998.

[For94] Giovanni Forni. Analytic destruction of invariant circles. Ergodic Theory Dynam. Systems, 14(2):267-298, 1994.

[FS93] Wendell H. Fleming and H. Mete Soner. Controlled Markov processes and viscosity solutions. Springer-Verlag, New York, 1993.

[Gom02] Diogo Aguiar Gomes. Viscosity solutions of Hamilton-Jacobi equations, and asymptotics for Hamiltonian systems. Calc. Var. Partial Differential Equations, 14(3):345-357, 2002. 
[LPV88] P. L. Lions, G. Papanicolao, and S. R. S. Varadhan. Homogeneization of Hamilton-Jacobi equations. Preliminary Version, 1988.

[Mat86] John Mather. A criterion for the nonexistence of invariant circles. Inst. Hautes Études Sci. Publ. Math., (63):153-204, 1986.

[Mat88] John N. Mather. Destruction of invariant circles. Ergodic Theory Dynam. Systems, 8*(Charles Conley Memorial Issue):199-214, 1988.

Diogo A. Gomes

Departamento de Matemática, Instituto Superior Técnico, Lisboa, 1049-001, Portugal

e-mail: dgomes@math.ist.utl.pt

\section{Enrico Valdinoci}

Dipartimento di Matematica, Università di Roma Tor Vergata, Roma, I-00133,

Italy

e-mail: valdinoci@mat.uniroma2.it 